\title{
The $\mathrm{kHz}$ QPOs as a probe of the X-ray color-color diagram and accretion-disk structure for the atoll source $4 \mathrm{U}$ 1728-34
}

\author{
De-Hua Wang ${ }^{1}$, Cheng-Min Zhang ${ }^{2,3,4}$, and Jin-Lu $\mathrm{Qu}^{5}$ \\ 1 School of Physics and Electronic Science, Guizhou Normal University, Guiyang 550001, PR China \\ e-mail: wangdh@gznu.edu.cn \\ 2 National Astronomical Observatories, Chinese Academy of Sciences, Beijing 100101, PR China \\ e-mail: zhangcm@bao.ac.cn \\ 3 School of Physical Sciences, University of Chinese Academy of Sciences, Beijing 101400, PR China \\ ${ }^{4}$ CAS Key Laboratory of FAST, Chinese Academy of Sciences, Beijing 100101, PR China \\ 5 Institute of High Energy Physics, Chinese Academy of Sciences, Beijing 100049, PR China
}

Received 16 January 2018 / Accepted 3 March 2018

\begin{abstract}
We have taken the $\mathrm{kHz}$ QPOs as a tool to probe the correlation between the tracks of X-ray color-color diagram (CCD) and magnetosphere-disk positions for the atoll source $4 \mathrm{U}$ 1728-34, based on the assumptions that the upper $\mathrm{kHz}$ QPO is ascribed to the Keplerian orbital motion and the neutron star (NS) magnetosphere is defined by the dipole magnetic field. We find that from the island to the banana state, the inner accretion disk gradually approaches the NS surface with the radius decreasing from $r \sim 33.0 \mathrm{~km}$ to $\sim 15.9 \mathrm{~km}$, corresponding to the magnetic field from $B(r) \sim 4.8 \times 10^{6} \mathrm{G}$ to $\sim 4.3 \times 10^{7} \mathrm{G}$. In addition, we note the characteristics of some particular radii of magnetosphere-disk $r$ are: firstly, the whole atoll shape of the CCD links the disk radius range of $\sim 15.9-33.0 \mathrm{~km}$, which is just located inside the corotation radius of $4 \mathrm{U} 1728-34 r_{\mathrm{co}}(\sim 34.4 \mathrm{~km})$, implying that the CCD shape is involved in the NS spin-up state. Secondly, the island and banana states of CCD correspond to the two particular boundaries: (I)near the corotation radius at $r \sim 27.2-33.0 \mathrm{~km}$, where the source lies in the island state; (II)near the NS surface at $r \sim 15.9-22.3 \mathrm{~km}$, where the source lies in both the island and banana states. Thirdly, the vertex of the atoll shape in CCD, where the radiation transition from the hard to soft photons occurs, is found to be near the NS surface at $r \sim 16.4 \mathrm{~km}$. The above results suggest that both the magnetic field and accretion environment are related to the CCD structure of atoll track, where the corotation radius and NS hard surface play the significant roles in the radiation distribution of atoll source.
\end{abstract}

Key words. X-rays: binaries - stars: neutron - stars: individual: 4U 1728-34 - accretion, accretion disks

\section{Introduction}

Neutron star low mass X-ray binaries (NS-LMXBs) can be classified as atoll and Z sources (Hasinger \& van der Klis 1989; van der Klis et al. 2006) according to the patterns they trace out in the X-ray color-color diagrams (CCDs). From the top to bottom in $\mathrm{CCD}$, the tracks of $\mathrm{Z}$ sources are called the horizontal, normal, and flare branches, while for atoll sources, they are called the extreme island, island, lower banana, and upper banana states. Initially, it is assumed that the mass-accretion rate of $\mathrm{Z}$ sources may increase monotonically along the branches (Hasinger 1990; Hasinger et al. 1990). However, this scenario cannot be used to interpret the "parallel tracks" phenomenon, in other words, the similar temporal and spectral properties are observed at the different luminosity (Méndez et al. 1999; Ford et al. 2000; van der Klis 2001). Alternatively, van der Klis (2001) proposed that the changes in the averaged accretion rate through the disk could be responsible for the motion along the $\mathrm{Z}$ track. In addition, the transition between the atoll and Z tracks has been observed in XTE $\mathrm{J} 1701-462$, which is explained as the influence by the changes in the accretion rate (Homan et al. 2007, 2010; Lin et al. 2009; Fridriksson et al. 2015). Other physical effects, for example, the NS magnetic field (Hasinger \& van der Klis 1989), have been proposed to interpret the difference between the two-subclass sources, however, there is no consensus as to their origin.
In both the atoll and $\mathrm{Z}$ sources the twin kilohertz quasiperiodic oscillations ( $\mathrm{kHz}$ QPOs, with lower $v_{1}$ and upper $v_{2}$ ) have been detected by Rossi X-ray Timing Explorer (RXTE; van der Klis et al. 2006), which show a non-linear relation between $v_{1}$ and $v_{2}$ (Belloni et al. 2002, 2005, 2007; Zhang et al. 2006) in the frequency range of $\simeq 100-1300 \mathrm{~Hz}$ (Wang et al. 2014). The frequencies of $\mathrm{kHz}$ QPOs are found to be correlated with other temporal and spectral features (e.g., Ford \& van der Klis 1998; Kaaret et al. 1998; Psaltis et al. 1999; Belloni et al. 2002; Méndez 2006), and they often increase monotonically along both the atoll tracks (e.g., van Straaten et al. 2000, 2003; Altamirano et al. 2008) and Z tracks (e.g., Wijnands et al. 1997, 1998; Homan et al. 2002; Jonker et al. 2002; Lin et al. 2012). It is suggested that $\mathrm{kHz}$ QPOs may reflect the orbital motion of the accreting matter at the inner accretion disk (Kluźniak et al. 1990; van der Klis 2000 and references therein; Zhang \& Wang 2013), which can be exploited to explore the physical environments in the strong gravitational field and strong magnetic field regions (Kluźniak et al. 1990; Abramowicz et al. 2003; Miller \& Miller 2015). Wang et al. $(2015,2017)$ analyzed the relations of the $\mathrm{kHz}$ QPO emission radii with the NS radius and corotation radius, suggesting that the emission of $\mathrm{kHz}$ QPOs may be affected by the NS hard surface and the spin-up environment near the corotation radius. 


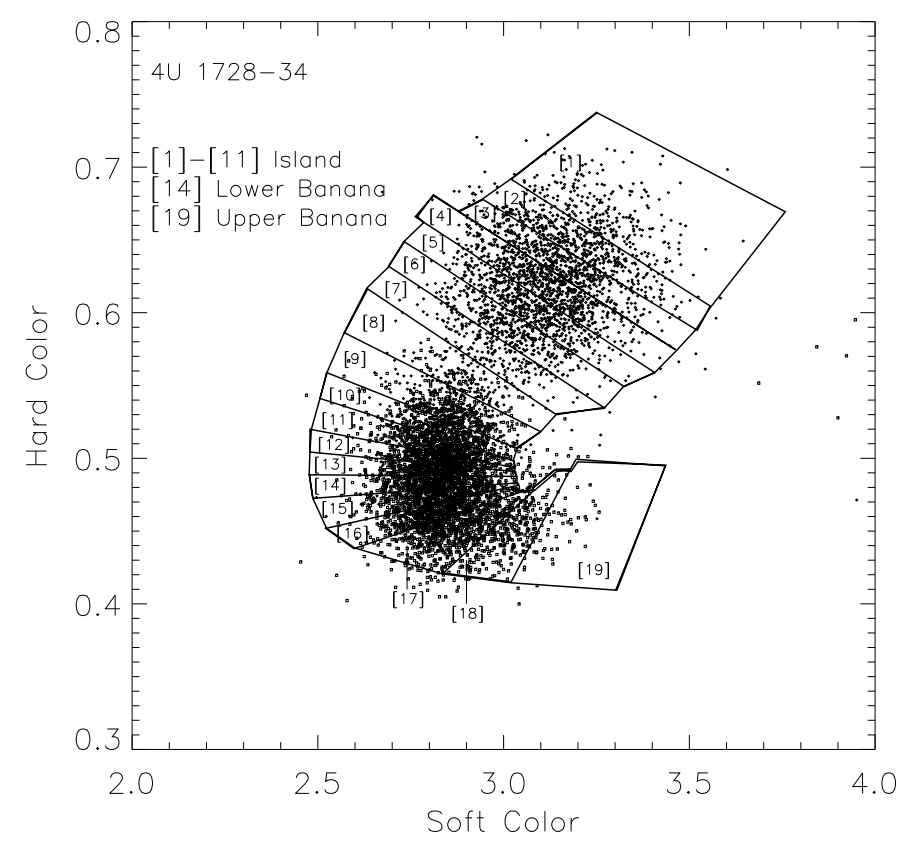

Fig. 1. X-ray color-color diagram of $4 \mathrm{U}$ 1728-34, where the soft and hard colors are defined as the ratio of the count rates in the bands $3.5-6.4 \mathrm{keV} / 2.0-3.5 \mathrm{keV}$ and $9.7-16 \mathrm{keV} / 6.4-9.7 \mathrm{keV}$, respectively. This labeled area of boxes indicate the intervals used to select the power spectra. The figure is reproduced with the same dataset of RXTE used by Di Salvo et al. (2001).

As yet, we do not understand why some atoll sources have been detected with the millisecond spin signals but the $\mathrm{Z}$ sources have not. In addition, among 30 NS-LMXBs with detected NS spin signals (Burderi \& Di Salvo 2013; van der Klis 2016), 12 sources have also been detected with twin $\mathrm{kHz}$ QPOs (Wang et al. 2017, 2018), including ten atoll sources. Particularly, in the atoll source 4U 1728-34 the X-ray burst oscillation has been observed with the frequency of $363 \mathrm{~Hz}$ (Strohmayer et al. 1996; Zhang et al. 2016), while it has also shown temporal variabilities on all timescales including kHz QPOs (e.g., Méndez \& van der Klis 1999; Di Salvo et al. 2001; van Straaten et al. 2002; Mukherjee \& Bhattacharyya 2012) and spectral evolution (e.g., Tarana et al. 2011; Mondal et al. 2017). The goal of this paper is to take the $\mathrm{kHz}$ QPOs as the tool to probe the relation between the X-ray CCD tracks of $4 \mathrm{U}$ 1728-34 and the magnetosphere-disk positions, and further investigate the effects of accretion environment and NS magnetic field on the formation of the atoll track.

The paper is organized as follows: In Sect. 2, we introduce the data of twin kHz QPOs and NS spin of 4U 1728-34. In Sect. 3 we probe the magnetosphere-disk positions and magnetic field strength along the atoll track. We have investigated the relation between the CCD shape and the particular disk radii, for example, NS radius and its corotation radius. In Sect. 4 we present the discussions and conclusions.

\section{Parameters of $4 \mathrm{U} \mathbf{1 7 2 8 - 3 4}$}

We collected the $\mathrm{kHz}$ QPO frequencies of 4U 1728-34 detected by Di Salvo et al. (2001) and the NS spin frequency detected by Strohmayer et al. (1996). For the kHz QPOs, Di Salvo et al. (2001) analyzed the RXTE data of 4U 1728-34 in 1996 between February 15 and March 1, 1996 on May 3, and 1997 between September 23 and October 1, with a total observation time of $\sim 456 \mathrm{ks}$. The authors plot the CCD by defining the soft and hard colors in CCD as the ratio of the count rate in the bands $3.5-6.4 \mathrm{keV} / 2.0-3.5 \mathrm{keV}$ and $9.7-16 \mathrm{keV} / 6.4-9.7 \mathrm{keV}$, respectively (see Fig. 1 in which we reproduce the CCD with the same dataset of RXTE used by Di Salvo et al. 2001). Then they divided the CCD into 19 intervals by the boxes as shown in Fig. 1 (see also Di Salvo et al. 2001), and further computed a power spectrum for each interval and fit the QPOs. The $\mathrm{kHz}$ QPOs have been detected in the intervals from [1] to [17] with the range of $v_{1} \sim 511-876.1 \mathrm{~Hz}$ and $v_{2} \sim 387-1158 \mathrm{~Hz}$ (see Table 1), where it can be seen that $v_{2}$ increases monotonically along the atoll track from the interval [1] to [17]. According to Di Salvo et al. (2001), the power spectra of interval [1]-[11] are typical of the island state of atoll sources, while the intervals [14]-[19] correspond to the lower and upper banana state, respectively. For the NS spin, Strohmayer et al. (1996) detected the burst oscillation of $4 \mathrm{U} 1728-34$ with the frequency of $363 \mathrm{~Hz}$ during the type I X-ray burst (see Table 1), which can be inferred as the NS spin frequency (Boutloukos \& Lamb 2008).

\section{Characteristic radii and magnetic field strength}

It is generally thought that the $\mathrm{kHz}$ QPOs reflect the motion of the accreting matter at the inner disk boundary around NS (Kluźniak et al. 1990; van der Klis 2000; van der Klis et al. 2006 and references therein). This implies the emission radius of the $\mathrm{kHz}$ QPOs may indicate the magnetosphere-disk radius. Furthermore, the $\mathrm{kHz}$ QPOs and color parameters at the different intervals in Fig. 1 are calculated by the same light curves (see Di Salvo et al. 2001 for the details), so the magnetospheredisk structures at various positions in X-ray CCD can be approximately inferred by the emission radius of $\mathrm{kHz}$ QPOs. In this paper, we take the detected kHz QPOs (Di Salvo et al. 2001) and NS spin (Strohmayer et al. 1996) of 4U 1728-34 to investigate the evolution of its magnetosphere-disk structure along the atoll track based on some assumptions (see the following equations), including the Keplerian orbital motion and magnetic dipole field.

\subsection{Magnetosphere-disk radius inferred by $\mathrm{kHz} Q P O s$}

Usually, the upper $\mathrm{kHz}$ QPO frequency $v_{2}$ is assumed as the Keplerian orbital frequency $v_{\mathrm{K}}$ of the accretion plasma. The emission radius of the $\mathrm{kHz}$ QPOs is explained as the magnetosphere-disk radius (e.g., Miller et al. 1998; Stella \& Vietri 1999; Stella et al. 1999; Lamb \& Miller 2001; Zhang 2004):

$v_{2}=v_{\mathrm{K}}=\sqrt{\frac{G M}{4 \pi^{2} r^{3}}}$,

where $G$ is the gravitational constant, $M$ is the NS mass and $r$ is the emission radius of the $\mathrm{kHz}$ QPOs referring to the NS center, that is, the magnetosphere-disk radius. By solving Eq. (1), $r$ can be derived as

$$
\begin{aligned}
r & =\left(\frac{G M}{4 \pi^{2}}\right)^{1 / 3} v_{2}^{-2 / 3} \\
& \approx 18.8(\mathrm{~km})\left(\frac{M}{1.6 M_{\odot}}\right)^{1 / 3}\left(\frac{v_{2}}{900 \mathrm{~Hz}}\right)^{-2 / 3} .
\end{aligned}
$$

We infer the magnetosphere-disk radius $r$ by Eq. (2) with the detected $v_{2}$ values in Table 1 and the assumed NS mass of $M \sim 1.6 M_{\odot}$ (on the average mass of the millisecond pulsars, see Zhang et al. 2011 and Özel \& Freire 2016). The results are 
Table 1. kHz QPOs and neutron star spin of 4U 1728-34 and the inferred parameters.

\begin{tabular}{|c|c|c|c|c|c|c|}
\hline $\begin{array}{l}\text { Interval } \\
\text { number }\end{array}$ & $\begin{array}{c}v_{1}^{a} \\
(\mathrm{~Hz})\end{array}$ & $\begin{array}{c}v_{2}^{b} \\
(\mathrm{~Hz})\end{array}$ & $\begin{array}{c}v_{\mathrm{s}}^{c} \\
(\mathrm{~Hz}) \\
\end{array}$ & $\begin{array}{c}r^{d} \\
(\mathrm{~km})\end{array}$ & $\begin{array}{c}B(r)^{e} \\
(\mathrm{G})\end{array}$ & $\begin{array}{c}r_{\mathrm{co}}^{f} \\
(\mathrm{~km})\end{array}$ \\
\hline$[1] \ldots$ & $\ldots$ & $387 \pm 18$ & 363 & 33.0 & $4.8 \times 10^{6}$ & 34.4 \\
\hline [2] ... & $\ldots$ & $397 \pm 14$ & & 32.4 & $5.1 \times 10^{6}$ & \\
\hline$[3] \ldots \ldots$ & $\ldots$ & $466 \pm 21$ & & 29.1 & $7.0 \times 10^{6}$ & \\
\hline$[4] \ldots \ldots$ & $\ldots$ & $497.8 \pm 5.3$ & & 27.9 & $8.0 \times 10^{6}$ & \\
\hline$[5] \ldots \ldots$ & $\ldots$ & $498.7 \pm 5.9$ & & 27.9 & $8.0 \times 10^{6}$ & \\
\hline$[6] \ldots \ldots$ & $\ldots$ & $517.3 \pm 8.1$ & & 27.2 & $8.6 \times 10^{6}$ & \\
\hline$[7] \ldots \ldots$ & $\ldots$ & $694.5 \pm 9.3$ & & 22.3 & $1.5 \times 10^{7}$ & \\
\hline$[8] \ldots \ldots$ & $\ldots$ & $729.0 \pm 3.5$ & & 21.6 & $1.7 \times 10^{7}$ & \\
\hline$[9] \ldots \ldots$ & $\ldots$ & $789.6 \pm 2.5$ & & 20.5 & $2.0 \times 10^{7}$ & \\
\hline$[10] \ldots \ldots$ & $511 \pm 17$ & $846.9 \pm 2.0$ & & 19.6 & $2.3 \times 10^{7}$ & \\
\hline$[11] \ldots \ldots$ & $559 \pm 11$ & $872.9 \pm 1.6$ & & 19.2 & $2.4 \times 10^{7}$ & \\
\hline$[12] \ldots \ldots$ & $599 \pm 14$ & $905.2 \pm 2.6$ & & 18.7 & $2.6 \times 10^{7}$ & \\
\hline$[13] \ldots \ldots$ & $673 \pm 10$ & $948.8 \pm 3.8$ & & 18.1 & $2.9 \times 10^{7}$ & \\
\hline$[14] \ldots \ldots$ & $750.9 \pm 3.7$ & $1054.7 \pm 8.7$ & & 16.9 & $3.6 \times 10^{7}$ & \\
\hline$[15] \ldots \ldots$ & $773.0 \pm 1.8$ & $1105.8 \pm 6.8$ & & 16.4 & $3.9 \times 10^{7}$ & \\
\hline$[16] \ldots .$. & $816.0 \pm 4.0$ & $1129 \pm 13$ & & 16.2 & $4.1 \times 10^{7}$ & \\
\hline$[17] \ldots \ldots$ & $876.1 \pm 2.6$ & $1158 \pm 18$ & & 15.9 & $4.3 \times 10^{7}$ & \\
\hline
\end{tabular}

Notes. ${ }^{(a)} v_{1}$-Frequency of the lower kHz QPO from Di Salvo et al. (2001). ${ }^{(b)} v_{2}$-Frequency of the upper kHz QPO from Di Salvo et al. (2001). ${ }^{(c)} v_{\mathrm{s}}$-Frequency of the neutron star spin from Strohmayer et al. (1996). ${ }^{(d)} r$-Emission radius of the kHz QPOs (the magnetosphere-disk radius) inferred by Eq. (2) with the assumed NS mass $M \sim 1.6 M_{\odot} \cdot{ }^{(e)} B(r)$-Magnetic field strength at the magnetosphere-disk radius inferred by Eq. (3) with the assumed NS surface magnetic field strength $B_{\mathrm{s}} \sim 10^{8} \mathrm{G}$ and NS radius $R \sim 12 \mathrm{~km} .{ }^{(f)} r_{\mathrm{co}}$-Corotation radius inferred by Eq. (4) with the assumed NS mass $M \sim 1.6 M_{\odot}$.

shown in Table 1, where it is noticed that $r$ lies in the range of $\sim 15.9-33.0 \mathrm{~km}$ and it decreases monotonically from the internal [1] $(r \sim 33.0 \mathrm{~km})$ to interval [17] $(r \sim 15.9 \mathrm{~km})$, which decreases by a factor of $\sim 50 \%$.

Figure $2 \mathrm{a}$ shows the plot of the magnetosphere-disk radius corresponding to each interval in CCD, where $r$ decreases monotonically along the atoll track, from the island state to the banana state. It should be also noticed that there is a radius gap between the interval [6] $(r \sim 27.2 \mathrm{~km})$ and interval [7] $(r \sim 22.3 \mathrm{~km})$ with few photons, and there is a vertex around the internal [15] ( $r \sim 16.4 \mathrm{~km}$ ) with the radiation changing from the hard photons dominated gradually to the soft photons dominated. For clarity, Fig. 3 shows the schematic diagram of the magnetospheredisk structures corresponding to the internal [1]-[17] in Fig. 2a, where the $\sim 5 \mathrm{~km}$ gap of the magnetosphere-disk radius between the internal [6] $(r \sim 27.2 \mathrm{~km})$ and [7] $(r \sim 22.3 \mathrm{~km})$ is obviously noticed.

\subsection{Magnetic field strength at the magnetosphere-disk radius}

In this paper the magnetic field of NS is assumed to be dipolar (Bhattacharya \& van den Heuvel 1991):

$$
\begin{aligned}
B(r) & =B_{\mathrm{s}}\left(\frac{R}{r}\right)^{3} \\
& \propto r^{-3},
\end{aligned}
$$

where $r$ is the radial distance referring to the NS center, $B_{\mathrm{s}}$ and $R$ are the surface magnetic field strength and the stellar radius of NS, respectively.

We inferred the magnetic field strength at the magnetosphere-disk radius, i.e., $B(r)$, by Eq. (3) with the inferred emission radius of $\mathrm{kHz}$ QPOs in Table 1 , as well as with the assumed NS surface magnetic field strength $B_{\mathrm{s}} \sim 10^{8} \mathrm{G}$ (Zhang \& Kojima 2006; Harding 2013; Mondal et al. 2017) and NS radius $R \sim 12 \mathrm{~km}$ (Shaposhnikov et al. 2003; Miller \& Miller 2015). The results are shown in Table 1 , where $B(r)$ ranges at $\sim 4.8 \times 10^{6} \mathrm{G}-4.3 \times 10^{7} \mathrm{G}$, and it increases monotonically from the internal [1] $\left(B(r) \sim 4.8 \times 10^{6} \mathrm{G}\right)$ to interval [17] $\left(B(r) \sim 4.3 \times 10^{7} \mathrm{G}\right)$, by nearly one order of magnitude.

Figure $2 \mathrm{~b}$ shows the plot of the $B(r)$ values at each interval in $\mathrm{CCD}$, which increases monotonically along the atoll track, from the island state to the banana state. We also note that the $B(r)$ value at the island-banana gap shown in Fig. 2a increases from $B(r) \sim 8.6 \times 10^{6} \mathrm{G}$ in the interval [6] to $B(r) \sim 1.5 \times 10^{7} \mathrm{G}$ in the interval [7], doubling its value. While the $B(r)$ value at the vertex of the atoll track around internal [15] in Fig. 2a is $B(r) \sim 3.9 \times 10^{7} \mathrm{G}$. For clarity, the schematic diagram of the magnetosphere-disk structure in Fig. 3 also shows the range of the $B(r)$ values of the interval [1]-[11] and interval [14], respectively.

\subsection{Corotation radius}

The corotation radius $r_{\mathrm{co}}$ in NS-LMXB is the radial distance referring to the NS center where the accretion plasma corotates with the NS magnetosphere, meaning that the Keplerian orbital frequency $v_{\mathrm{K}}$ there equals the NS spin frequency $v_{\mathrm{S}}$ (Bhattacharya \& van den Heuvel 1991). Setting $v_{\mathrm{K}}=v_{\mathrm{s}}$ in Eq. (1), $r_{\text {co }}$ can be derived as

$$
\begin{aligned}
r_{\mathrm{co}} & =\left(\frac{G M}{4 \pi^{2}}\right)^{1 / 3} v_{\mathrm{s}}^{-2 / 3} \\
& \approx 34.4(\mathrm{~km})\left(\frac{M}{1.6 M_{\odot}}\right)^{1 / 3}\left(\frac{v_{\mathrm{s}}}{363 \mathrm{~Hz}}\right)^{-2 / 3} .
\end{aligned}
$$



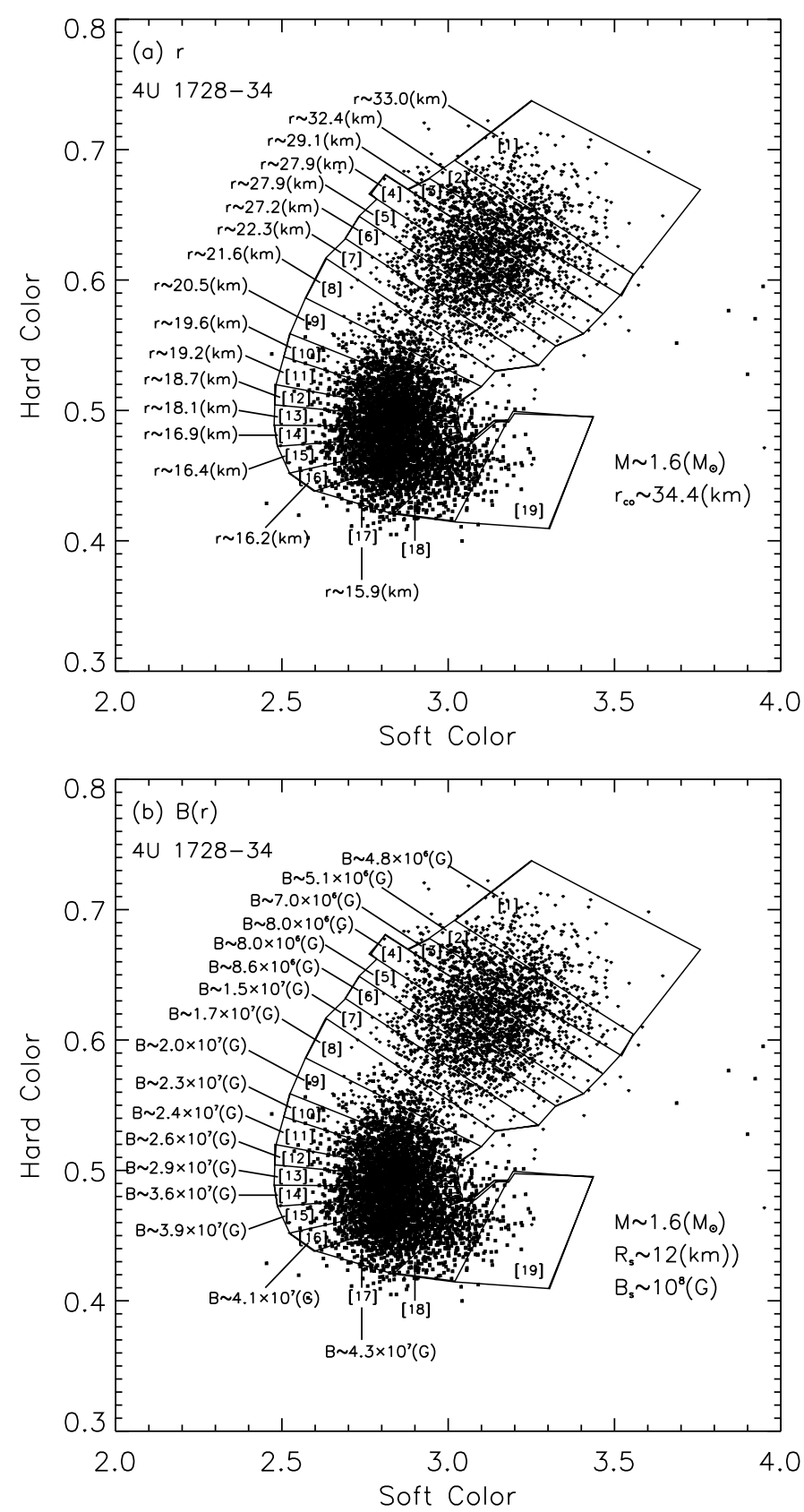

Fig. 2. Panel $a$ : magnetosphere-disk radius $r$ at various positions (interval [1]-[17]) in X-ray color-color diagram. Panel b: similar to (panel a), but for the magnetic field strength $B$ at $r$. The observed CCD points of 4 U 1728-34 are taken from the paper by Di Salvo et al. (2001). The figure is reproduced with the RXTE dataset used by Di Salvo et al. (2001).

We infer the corotation radius of $4 \mathrm{U} 1728-34$ by Eq. (4) with the inferred NS spin frequency of $v_{\mathrm{s}} \sim 363 \mathrm{~Hz}$ (Strohmayer et al. 1996) and the assumed NS mass of $M \sim 1.6 M_{\odot}$ (Zhang et al. 2011; Özel \& Freire 2016), and obtain $r_{\text {co }} \sim 34.4 \mathrm{~km}$ (see Table 1). We note that all the magnetosphere-disk radii $(r \sim 15.9-33.0 \mathrm{~km}$, see Table 1$)$ of the source inferred by the $\mathrm{kHz}$ QPOs are smaller than its corotation radius (see also Fig. 3).

\section{Discussions and conclusions}

We assumed that the upper $\mathrm{kHz}$ QPO is ascribed to the Keplerian orbital motion and the NS magnetosphere is defined

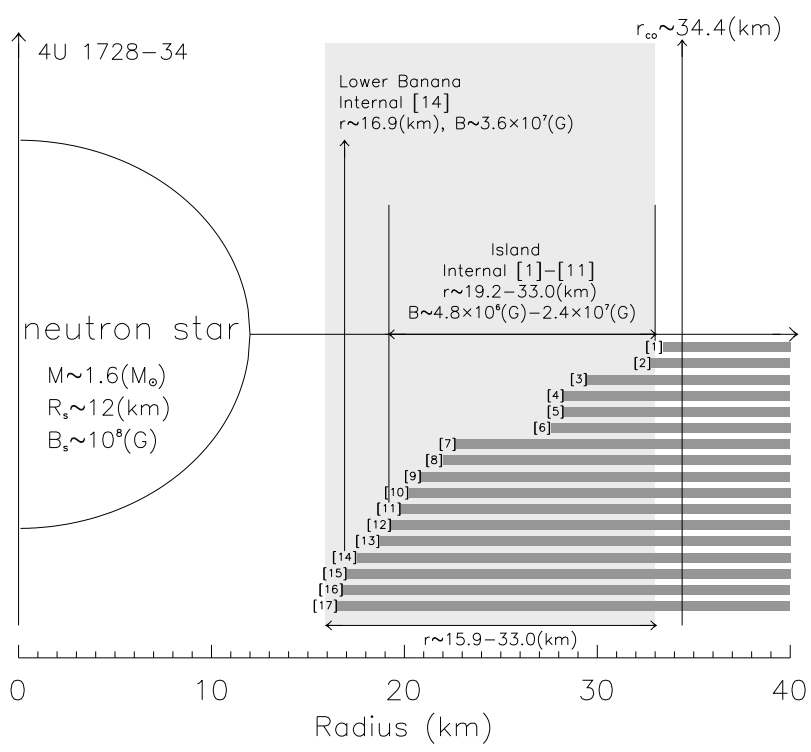

Fig. 3. Schematic diagram of the magnetosphere-disk structures for various positions in X-ray color-color diagram, where the number [1]-[17] are corresponding to the internals as described in Fig. 2. The position of the corotation radius and the ranges of the magnetic field strength are also shown.

by the dipole magnetic field, as well as the detected $\mathrm{kHz}$ QPOs from Di Salvo et al. (2001) and NS spin $(363 \mathrm{~Hz})$ inferred by the type I X-ray burst oscillation (Strohmayer et al. 1996). Based on these assumptions, we investigated the evolution of the magnetosphere-disk structure of $4 \mathrm{U} 1728-34$ along the atoll track. We find that the magnetosphere-disk radius of the source decreases monotonically from the island state to the banana state in CCD of the source. Below we summarize the details of the discussions and conclusions.

As the source evolves from the island state to the banana state, its magnetosphere-disk radius decreases nearly by half (see Table 1 and Fig. 2a), while the accretion disk gradually enters the stronger magnetic field region with the strength increasing by a factor of 9 (see Table 1 and Fig. 2b). We note that these results rely on the simplistic assumptions of the Keplerian motion and magnetic dipole field (see Eqs. (1)-(4)). However, the reality of the problem is much more complex: due to the viscous of the accretion plasma and its interaction with the NS magnetic field, the orbital velocity of the plasma at a certain radius may be less than the Keplerian orbital velocity there, and Eq. (1) should be revised as

$v_{2}=\xi v_{\mathrm{K}}=\xi \sqrt{\frac{G M}{4 \pi^{2} r^{3}}}$,

where $0<\xi \leq 1$. The value of $\xi$ can be estimated as follows: the observed maximal $\mathrm{kHz}$ QPO frequency of $4 \mathrm{U} 1728-34$ is $\sim 1200 \mathrm{~Hz}$ (Migliari et al. 2003), inferring the Keplerian orbital radius of $r \sim 15.5 \mathrm{~km}$ with the assumed NS mass of $1.6 M_{\odot}$. By assuming the actual emission radius should be less than $15.5 \mathrm{~km}$, for example, $r \sim 15 \mathrm{~km}$, we can infer the value of $\xi$ to be

$\xi=95 \%\left(\frac{v_{2}}{1200 \mathrm{~Hz}}\right)\left(\frac{M}{1.6 M_{\odot}}\right)^{-1 / 2}\left(\frac{r}{15 \mathrm{~km}}\right)^{3 / 2}$.

If $\xi$ is a constant, the variation of the magnetosphere-disk radius from the internal [1] to [17] can be derived as $r_{[1]} / r_{[17]} \propto$ $\left(v_{2[1]} / v_{2[17]}\right)^{-2 / 3} \sim(387 / 1158)^{-2 / 3} \approx 2.1$. Furthermore, in vacuum, the dipole field lines are poloidal with $B \propto r^{-3}$, which 
increases from the internal [1] to [17] by a factor of $B_{[17]} / B_{[1]} \propto$ $\left(r_{[1]} / r_{[17]}\right)^{3} \sim(2.1)^{3} \approx 9.2$. In the presence of the accretion disk, the field lines are initially "frozen-in" to the orbiting plasma, which hence are sheared in the $\phi$ direction (Ghosh \& Lamb 1978; Shapiro \& Teukolsky 1983). This shearing generates a sizeable $B_{\phi}$ component, causing the magnetic field strength around the accretion disk may be stronger than the dipole field, as a general form,

$B(r)=B_{\mathrm{s}}\left(\frac{R}{r}\right)^{\alpha}$,

with $\alpha \geq 3$. If we assume $\alpha \sim 3.5$, the magnetic field strength from the internal [1] to [17] should increase by a factor of $B_{[17]} / B_{[1]} \propto\left(r_{[1]} / r_{[17]}\right)^{3.5} \sim(2.1)^{3.5} \approx 13.4$. However, these corrections do not obviously affect the conclusions that, as the evolution of the atoll track of $4 \mathrm{U}$ 1728-34, the accretion disk moves toward the NS surface and gradually enters the stronger magnetic field region.

It can be seen from Fig. 2a that the whole atoll track of $4 \mathrm{U} 1728-34$ is formed when the magnetosphere-disk radii are smaller than the corotation radius, i.e., $r / r_{\mathrm{co}} \propto\left(v_{\mathrm{s}} / v_{2}\right)^{2 / 3} \leq$ $(363 \mathrm{~Hz} / 387 \mathrm{~Hz})^{2 / 3} \sim 0.95$. The accretion plasma corotates with the magnetosphere at $r_{\mathrm{co}}$, while according to the expression of the Keplerian orbital velocity:

$$
\begin{aligned}
v & =\left(2 \pi G M v_{2}\right)^{1 / 3} \\
& \propto v_{2}^{1 / 3},
\end{aligned}
$$

as the accretion disk moves toward the NS surface from the internal [1] to [17], the orbital velocity of the plasma increases gradually by a factor of $\left(1158^{1 / 3}-387^{1 / 3}\right) / 387^{1 / 3} \sim 44 \%$. The large velocity difference $\Delta v \propto v_{2}^{1 / 3}-v_{\mathrm{s}}^{1 / 3}$ between the magnetosphere and the accretion disk may induce the strong interaction and release huge energy (Wang et al. 2017). We suspect that this process can provide the particular physical environment to soften the photons, which causes the evolution of the atoll track from island state to banana state.

Figure 3 shows a position gap around $r \sim 27.2-22.3 \mathrm{~km}$ between the internal [6] and [7] (see Table 1), which can be used to classify the magnetosphere-disk structures into two categories: (I)internal [1]-[6] with $r \sim 27.2-33.0 \mathrm{~km}$, where the source is in the island state and $r$ is near the corotation radius $\left(r_{\text {co }} \sim 34.4 \mathrm{~km}\right.$, see Table 1); (II)internal [7]-[17] with $r \sim$ $15.9-22.3 \mathrm{~km}$, where the source is in the island state and banana state with $r$ approaching the NS surface. This "gap" may be due to the lack of the observational time (Zhang et al. 2016). However, we also suggest that the corotation radius and NS surface may be the two characteristic boundaries for the atoll track evolution: the corotation radius may be a soft boundary, and we guess the common rotation frequency of accretion plasma and magnetosphere may induce some type of resonance effect and release huge energy. Furthermore, the radiation around this position may be hard-photon dominated, as shown in the island state (see Fig. 2). While the NS surface is a hard boundary, and when the accretion plasma collide on this hard surface or on the local strong magnetic field (Zhang \& Kojima 2006), the source may release huge energy and produce much soft photons. This guess is supported by the fact that there is a vertex around the internal [15] in CCD, where the radiation varies from the hard photons dominated gradually to the soft photons dominated. We note from Fig. 3 that the inner radius corresponding to the vertex is quite near the NS surface $(r \sim 16.4 \mathrm{~km})$, and the distance difference of the inner radii between internal [15] $(r \sim 16.4 \mathrm{~km})$ and [17] $(r \sim 15.9 \mathrm{~km})$ is as short as $\sim 0.5 \mathrm{~km}$.
The parallel tracks in the $\mathrm{kHz}$ QPO evolution have been observed in 4U 1728-34 (Méndez et al. 2001; van der Klis 2001), in other words, the same $\mathrm{kHz}$ QPO frequency could correspond to at least two different luminosities (or count rates) and colors during different observations. This phenomenon may deviate from our conclusion, since taking a different track may end up with different results for the same frequency. Here we have adopted the double accretion rates suggested by van der Klis (2001) to explain this phenomenon of parallel lines. As such, the luminosity of the source depends on the total mass accretion rate $\dot{M}_{\text {tot }}$, which comes from the disk accretion $\dot{M}_{\mathrm{d}}$ and the radial accretion $\dot{M}_{\text {rad }}$ (see also Pan et al. 2016):

$\dot{M}_{\text {tot }}=\dot{M}_{\mathrm{d}}+\dot{M}_{\mathrm{rad}}$.

The QPOs are likely to origin from the accretion disk (van der Klis 2001) due to the quasi-periodic property and are affected by $\dot{M}_{\mathrm{d}}$. As $\dot{M}_{\mathrm{d}}$ increases, the accretion disk moves toward the NS surface, accompanied by the increasing of the kHz QPO frequency and the formation of the atoll track. In addition, the radial accretion may exist, since the accretion disk is thickened by the radiation pressure as it moves toward the NS surface, which causes an approximate spherical accretion in the region that is smaller than the corotation radius of $r_{\text {co }} \sim 34.4 \mathrm{~km}$. The different radial accretion and the disk accretion rates can account for the same magnetosphere-disk radii, which can account for the same $\mathrm{kHz}$ QPOs, while they produce the different luminosity. In other words, $\dot{M}_{\text {rad }}$ can modulate both the luminosity and the colors of the source, which modulates a same magnetosphere-disk radius as a $\dot{M}_{\mathrm{d}}$ does, thus a phenomenon of the parallel tracks occurs.

Based on the above discussions, we suggest that the atoll track in CCD of $4 \mathrm{U} 1728-34$ may correspond to the particular magnetosphere-disk boundary, where the corotation radius and NS hard surface could play a significant role in the different radiation processes. In addition, it seems that the magnetic field strength and spin-up state of NS may also contribute to the radiation composition in the CCD. As a further exploration, however, similar investigations in other atoll sources and $\mathrm{Z}$ sources are needed to test whether these phenomena are common to all NS-LMXBs. If confirmed, these results will help to understand the formation mechanism of the atoll CCD track, to probe the accretion environment and the magnetic field structure around NS, and also to test the presence mechanism of $\mathrm{kHz}$ QPOs.

Acknowledgements. This work is supported by the National Program on Key Research and Development Project (Grant No. 2016YFA0400803), the National Natural Science Foundation of China (Grant No. 11703003, No. 11673023 and No. U1731238).

\section{References}

Abramowicz, M. A., Karas, V., Kluźniak, W., Lee, W. H., \& Rebusco, P. 2003, PASJ, 55, 467

Altamirano, D., van der Klis, M., Meńdez, M., et al. 2008, ApJ, 685, 436

Belloni, T., Psaltis, D., \& van der Klis, M. 2002, ApJ, 572, 392

Belloni, T., Méndez, M., \& Homan, J. 2005, A\&A, 437, 209

Belloni, T., Méndez, M., \& Homan, J. 2007, MNRAS, 376, 1133

Bhattacharya, D., \& van den Heuvel, E. P. J. 1991, Phys. Rep., 203, 1

Boutloukos, S., \& Lamb, F. K. 2008, in 40 Years of Pulsars: Millisecond Pulsars, Magnetars, and More, eds. C. G. Bassa, et al. (Melville, NY: Am. Inst. Phys.), AIP Conf. Ser., 983, 533

Burderi, L., \& Di Salvo, T. 2013, Mem. Soc. Astron. It., 84, 117

Di Salvo, T., Méndez, M., van der Klis, M., Ford, E., \& Robba, N. R. 2001, ApJ, 546, 1107

Ford, E. C., \& van der Klis, M. 1998, ApJ, 506, L39

Ford, E. C., van der Klis, M., Méndez, M., et al. 2000, ApJ, 537, 368 Fridriksson, J. K., Homan, J., \& Remillard, R. A. 2015, ApJ, 809, 52 
Ghosh, P., \& Lamb, F. K. 1978, ApJ, 223, L83

Harding, A. K. 2013, Front. Phys., 8, 679

Hasinger, G. 1990, Rev. Mod. Astron., 3, 60

Hasinger, G., \& van der Klis, M. 1989, A\&A, 225, 79

Hasinger, G., van der Klis, M., Ebisawa, K., Dotani, T., \& Mitsuda, K. 1990, A\&A, 235, 131

Homan, J., van der Klis, M., Jonker, P. G., et al. 2002, ApJ, 568, 878

Homan, J., van der Klis, M., Wijnands, R., et al. 2007, ApJ, 656, 420

Homan, J., van der Klis, M., Fridriksson, J. K., et al. 2010, ApJ, 719, 201

Jonker, P. G., van der Klis, K., Homan, J., et al. 2002, MNRAS, 333, 665

Kaaret, P., Yu, W., Ford, E. C., \& Zhang, S. N. 1998, ApJ, 497, L93

Kluźniak, W., Michelson, P., \& Wagoner, R. V. 1990, ApJ, 358, 538

Lamb, F. K., \& Miller, M. C. 2001, ApJ, 554, 1210

Lin, D. C., Remillard, R. A., \& Homan, J. 2009, ApJ, 696, 1257

Lin, D. C., Remillard, R. A., Homan, J., \& Barret, D. 2012, ApJ, 756, 34

Méndez, M. 2006, MNRAS, 371, 1925

Méndez, M., \& van der Klis, M. 1999, ApJ, 517, L51

Méndez, M., van der Klis, K., Ford, E. C., Wijnands, R., \& van Paradijs, J. 1999, ApJ, 511, L49

Méndez, M., van der Klis, K., \& Ford, E. C. 2001, ApJ, 561, 1016

Migliari, S., van der Klis, M., \& Fender, R. P. 2003, MNRAS, 345, L35

Miller, M. C., \& Miller, J. M. 2015, Phys. Rep., 548, 1

Miller, M. C., Lamb, F. K., \& Psaltis, D. 1998, ApJ, 508, 791

Mondal, A. S., Pahari, M., Dewangan, G. C., Misra, R., \& Raychaudhuri, B. 2017, MNRAS, 466, 4991

Mukherjee, A., \& Bhattacharyya, S. 2012, ApJ, 756, 55

Özel, F., \& Freire, P. 2016, ARA\&A, 54, 401

Pan, Y. Y., Song, L. M., Zhang, C. M., \& Tong, H. 2016, MNRAS, 461, 2

Psaltis, D., Belloni, T., \& van der Klis, M. 1999, ApJ, 520, 262

Shaposhnikov, N., Titarchuk, L., \& Haberl, F. 2003, ApJ, 593, 35

Shapiro, S. L., \& Teukolsky, S. A. 1983, Black Holes, White Dwarfs, and Neutron Stars: The Physics of Compact Objects (New York: Wiley Interscience)
Stella, L., \& Vietri, M. 1999, Phys. Rev. Lett., 82, 17

Stella, L., Vietri, M., \& Morsink, S. M. 1999, ApJ, 524, L63

Strohmayer, T. E., Zhang, W., Swank, J. H., et al. 1996, ApJ, 469, L9

Tarana, A., Belloni, T., Bazzano, A., Méndez, M., \& Ubertini, P. 2011, MNRAS, 416,873

van der Klis, M. 2000, ARA\&A, 38, 717

van der Klis, M. 2001, ApJ, 561, 943

van der Klis, M. 2006, in Compact Stellar X-Ray Sources, eds. W. H. G. Lewin, \& M. van der Klis (Cambridge: Cambridge Univ. Press), 39

van der Klis, M. 2016, in Invited talk in European Week of Astronomy and Space Science, Timing Low-Mass X-Ray Binaries and Accreting Millisecond Pulsars, 4-8 July 2016, Athens, Greece

van Straaten, S., Ford, E. C., van der Klis, M., Méndez, M., \& Kaaret, P. 2000, ApJ, 540, 1049

van Straaten, S., van der Klis, M., Di Salvo, T., \& Belloni, T. 2002, ApJ, 568, 912

van Straaten, S., van der Klis, M., \& Méndez, M. 2003, ApJ, 596, 1155

Wang, D. H., Chen, L., Zhang, C. M., \& Qu, J. L. 2014, Astron. Nachr., 335, 168

Wang, D. H., Chen, L., Zhang, C. M., et al. 2015, MNRAS, 454, 1231

Wang, D. H., Zhang, C. M., Lei, Y. J., et al. 2017, MNRAS, 1111, 1117

Wang, D. H., Zhang, C. M., Qu, J. L., \& Yang, Y. Y. 2018, MNRAS, 473, 4862

Wijnands, R., Homan, J., van der Klis, M., et al. 1997, ApJ, 490, L157

Wijnands, R., Meńdez, M., van der Klis, M., et al. 1998, ApJ, 504, L35

Zhang, C. M. 2004, A\&A, 423, 401

Zhang, C. M., \& Kojima, Y. 2006, MNRAS, 366, 137

Zhang, C. M., Yin, H. X., Zhao, Y. H., Zhang, F., \& Song, L. M. 2006, MNRAS, 366, 1373

Zhang, C. M., Wang, J., Zhao, Y. H., et al. 2011, A\&A, 527, A83

Zhang, C. M., \& Wang, D. H. 2013, in Feeding Compact Objects: Accretion on All Scales, eds. C. M. Zhang, T. Belloni, M. Mendez, et al. (Cambridge: Cambridge University Press), Proceedings of IAU Symp., 290, 381

Zhang, G., Méndez, M., Zamfir, M., \& Cumming, A. 2016, MNRAS, 455, 2004 